\title{
The prognostic factors influencing overall survival in uterine cervical cancer with brain metastasis
}

\author{
Hyera Kim, Kang Kook Lee, Mi Hwa Heo, and Jin Young Kim
}

Division of Hematology and Oncology, Department of Internal Medicine, Keimyung University Dongsan Medical Center, Daegu, Korea

Received: February 6, 2018 Revised : March 19, 2018 Accepted: May 2, 2018

\section{Correspondence to}

Hyera Kim, M.D.

Division of Hematology and Oncology, Department of Internal Medicine, Keimyung University Dongsan Medical Center, 56 Dalseong-ro, Jung-gu, Daegu 41931, Korea

Tel: $+82-53-250-8434$

Fax: +82-53-250-7434

E-mail:kheyra@dsmc.or.kr
Background/Aims: The occurrence of brain metastasis (BM) has increased due to improved overall survival (OS) in uterine cervical cancer. However, research about prognostic factors and therapeutic guidelines for BM in uterine cervical cancer remains scarce due to the rarity of $\mathrm{BM}$ in this type of cancer. The present study evaluated the clinical characteristics and prognostic factors influencing OS in patients with BM from uterine cervical cancer.

Methods: A total of 19 BM patients of uterine cervical cancer were analyzed retrospectively from January 1995 to December 2016.

Results: The median and mean OS of all patients was 9.6 and 15.4 months. Treatment (vs. palliative care, $p<0.001$ ), fewer than three regimens of chemotherapy before BM (vs. $\geq 3, p<0.013$ ), and chemotherapy after BM (vs. absence, $p<0.001$ ) significantly increased the OS time. The Karnofsky performance status $\geq 70$ (vs. < $70, p=0.213$ ), single BM (vs. multiple BM, $p=0.157$ ), and small cell carcinoma (vs. others, $p=0.351$ ) had numerically higher OS than others. Dual therapy (vs. single therapy, $p=0.182$; vs. no therapy, $p=0.076$ ) were associated with a longer OS time, but the difference did not reach statistical significance. In addition, the graded prognostic assessment (GPA) appeared to be a better prognostic tool than the recursive partitioning analysis.

Conclusions: The results of the present study suggest active multimodal treatment including neurosurgery, radiotherapy, and chemotherapy for BM of uterine cervical cancer with single BM, good performance status, histology of small cell carcinoma, and a better GPA.

Keywords: Brain metastasis; Uterine cervical neoplasms; Prognosis

\section{INTRODUCTION}

In 2012, uterine cervical cancer was the fourth most common cancer and the fourth most frequent cause of cancer-related death among women worldwide [1]. According to the Korea Central Cancer Registry, uterine cervical cancer was the fifth most prevalent cancer in Korean women in 2015 [2]. In uterine cervical cancer, com- mon sites of distant metastasis include the liver, lung, and bone. While brain metastasis (BM) is the most common intracranial tumor, BM from uterine cervical cancer is extremely rare, with its incidence rate amounting to mere $0.57 \%$ [3]. Despite these low rates, the occurrence of BM appears to have increased in recent years [4]. This increase may be due to the increased overall survival (OS) from its early detection and improved treatment [5]. 
When BM develops in patients with uterine cervical cancer, it is an indicator of a poor prognosis, with the median survival ranging from 1 to 8 months [6]. BM is usually treated with multimodal therapy using a combination of neurosurgery, radiotherapy, and chemotherapy [7]. In a previous study, patients undergoing this combination of therapies had a longer survival time than those undergoing single therapy, and clarification of the clinical characteristics of patients who would benefit from multimodal therapy was reported to be an important issue [8]. However, due to the rarity of BM from uterine cervical cancer, there are no optimal therapeutic guidelines on or prognostic factors for this condition.

Numerous studies have analyzed prognostic factors and recommended prognostic scoring systems for BM [9]. The Radiation Therapy Oncology Group recommends that the treatment choice for BM from extracranial primary cancer should be based on age, Karnofsky performance status (KPS), control of primary tumor, and the status of extracranial disease [10]. However, this recursive partitioning analysis (RPA) does not include the number of BM in the prognostic score. In 2007, a new scoring system called the graded prognostic assessment (GPA) was proposed; this system included the number of BM [11]. However, although both the older and the new scoring systems have commonly used as prognostic indexes in clinical practice, the data on evaluating and comparing these systems for the patients with BM from uterine cervical cancer are scarce.

To fill this gap, in the present study, we collected data on uterine cervical cancer with BM over a 22-year period from a single institute and evaluated the clinical characteristics and prognostic factors influencing OS in patients.

\section{METHODS}

The medical records of 2,774 patients with uterine cervical cancer who had been treated at Keimyung University Dongsan Medical Center from January 1995 to December 2016 were retrospectively reviewed. Among them, 19 patients had a pathological diagnosis of primary uterine cervical cancer and were clinically or radiologically diagnosed with BM from uterine cervical cancer. The patients with other active malignancies, or a central nerve
Table 1. Definition of the graded prognostic assessment for patients with brain metastasis

\begin{tabular}{lccc}
\hline \multirow{2}{*}{ Prognostic factors } & \multicolumn{3}{c}{ Score } \\
\cline { 2 - 4 } & $\geq 60$ & 0.5 & 1.0 \\
\hline Age & $<70$ & $70-80$ & $90-100$ \\
KPS & $>3$ & $2-3$ & 1 \\
No. of BM & Present & - & None \\
Extracranial metastasis &
\end{tabular}

KPS, Karnofsky performance status; BM, brain metastasis.

system disease unrelated to BM, were excluded. This study was approved by the Institutional Review Board of the Keimyung University Dongsan Medical Center (DSMC-2018-01-045), which waived the requirement for written informed consent because of the retrospective nature of the present study.

The clinical data included the date of birth, the Charlson comorbidity index, histology, initial the International Federation of Gynecology and Obstetrics (FIGO) stage, date of the original cancer diagnosis and presence of $\mathrm{BM}$, the number of chemotherapy regimens before $\mathrm{BM}$, whether the primary lesion was controlled at BM diagnosis, date of death or the last follow-up visit, KPS at $\mathrm{BM}$ diagnosis, number, site, and maximum size of $\mathrm{BM}$, and whether extracranial metastases were present. All local and systemic therapy modalities, such as palliative care, neurosurgery, radiotherapy for the brain, and chemotherapy after the diagnosis of BM, were analyzed. Palliative care was defined as symptomatic care without neurosurgery, radiotherapy, or chemotherapy. According to the classification method proposed in Gaspar et al. [10], patients with KPS $\geq 70$, aged below 65 years old, controlled primary and no other systemic metastasis were grouped in RPA class I. On the other hand, RPA class III included all the patients with KPS below 70, and the remaining patients were grouped into RPA class II. In the GPA, each of the factors such as age, KPS, extracranial metastasis, and number of BM were given a score of 0 , 0.5, or 1.0, and GPA was calculated as the sum score of all four factors (Table 1) [11].

According to the Kaplan-Meier method, the OS was calculated from the date of diagnosis of BM to death of any reason or the last day of follow-up and then analyzed based on the log-rank test. The level of critical significance was assigned at $p<0.05$. Statistical data were 


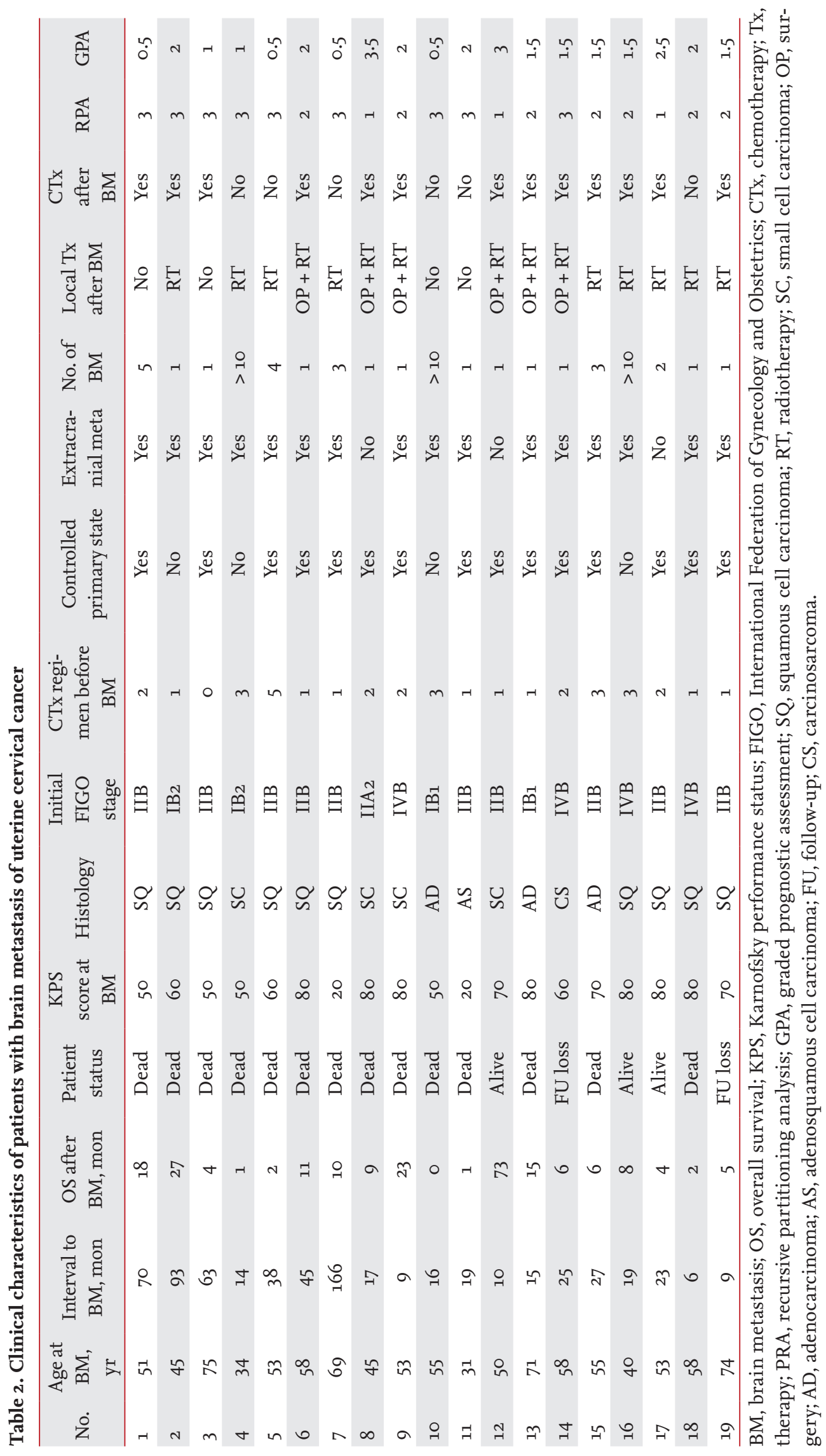




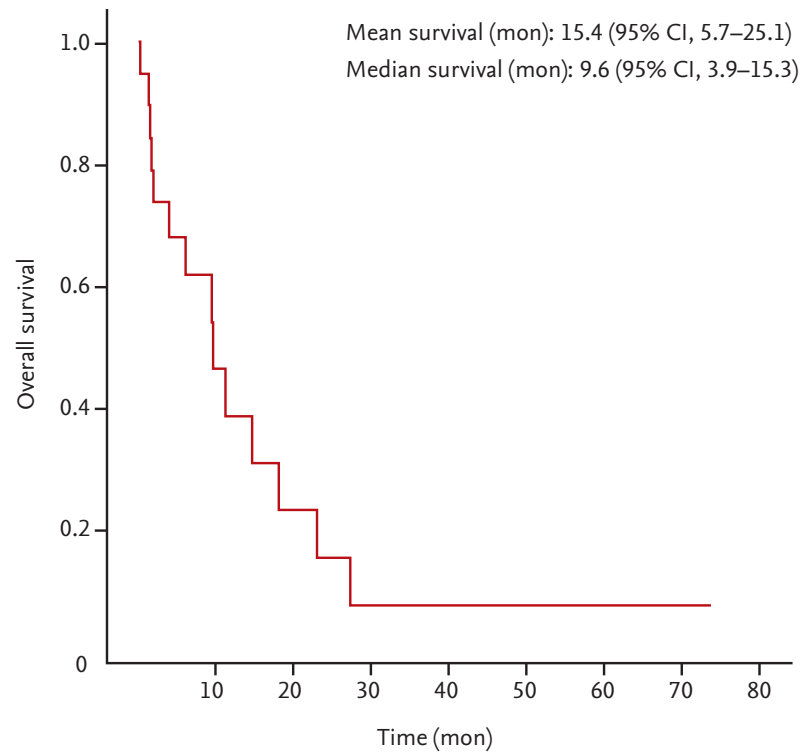

Figure 1. The Kaplan-Meier survival curve in patients with brain metastasis from uterine cervical cancer. CI, confidence interval. analyzed using the IBM SPSS Statistics for Windows version 19.0 (IBM Co., Armonk, NY, USA).

\section{RESULTS}

\section{Patient characteristics}

The incidence rate of BM from initial or recurrent uterine cervical cancer in our institution was $0.68 \%(19 / 2,774)$. Patient characteristics are summarized in Table 2. The mean age at diagnosis of BM was 54.1 years. KPS was < 70 in nine patients (47.4\%). Concerning the initial FIGO stage, four patients (21.1\%) had stage I, 11 (57.9\%) had stage II, and four (21.1\%) had stage IV. The histology of uterine cervical cancer were squamous cell carcinoma in $10(52.6 \%)$, small cell carcinoma in four $(21.1 \%)$, and adenocarcinoma in three patients (15.8\%). Five patients (26.3\%) were heavily treated with more than three regi-
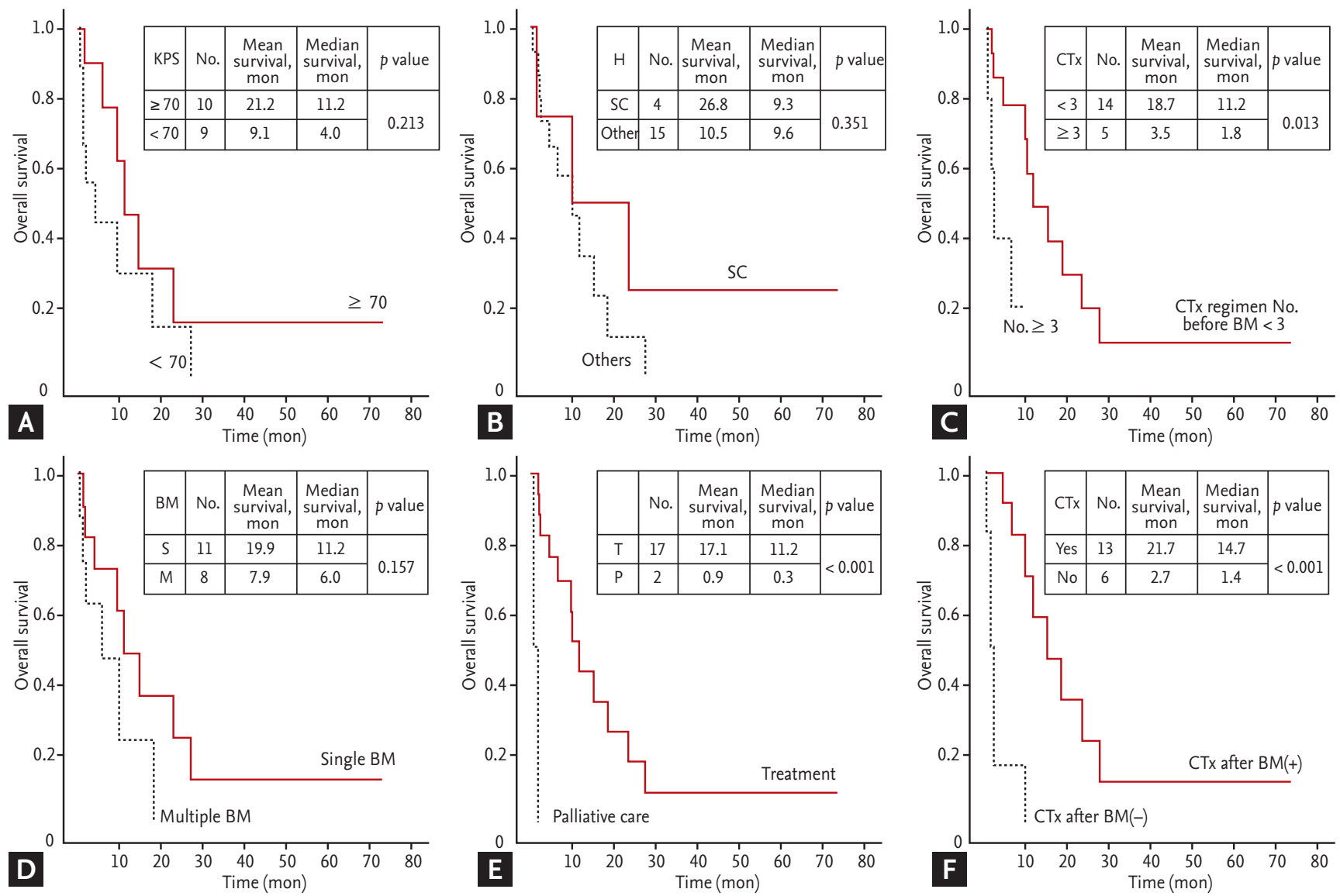

Figure 2. The Kaplan-Meier survival curves in patients with brain metastasis (BM) from uterine cervical cancer based on prognostic factors influencing the survival time: (A) the Karnofsky performance score (KPS, $\geq 70$ vs. < 70), (B) histology (small cell carcinoma [SC] vs. others), (C) the number of chemotherapy (CTx) regimens before BM ( $\geq 3$ vs. $<3$ ), (D) the number of BM (single vs. multiple), (E) treatments for BM (treatment vs. palliative care), (F) CTx after BM (presence vs. absence). H, histology. 
mens of chemotherapy before BM. The primary tumor was controlled in 15 patients (78.9\%), and 16 patients (84.2\%) had extracranial metastasis.

The median and mean time from diagnosis of the primary uterine cervical cancer to BM amounted to 19.3 and 35.9 months, respectively. BM was detected in all patients metachronously. Eleven (57.9\%), four (21.1\%), and four $(21.1 \%)$ patients had a solitary, 2 to 4 , and $\geq 5$ lesions, respectively. The mean maximum diameter of $\mathrm{BM}$ was $31.6 \mathrm{~mm}$, and 10 patients (52.6\%) had more than $30 \mathrm{~mm}$ of mass. Thirteen (68.4\%) were located in the supratentorial area; two (10.5\%) in the infratentorial area; and four $(21.1 \%)$ in both areas. Eleven patients $(57.9 \%)$ had neurologic symptoms (e.g., limb weakness and mental change), and eight (42.1\%) had a headache. According to the RPA, three patients (15.8\%) were categorized as RPA class I, and nine patients (47.4\%) were categorized as RPA class III for their KPS score below 70. Furthermore, six patients (31.6\%) had a GPA score of o to 1, and three patients $(15.8 \%)$ had a GPA score 2.5 or above.

Two patients underwent only palliative care, while the remaining 17 were treated with local and systemic treatment such as neurosurgery, radiotherapy including whole brain radiotherapy and stereotactic radiosurgery, or chemotherapy. Fifteen patients (78.9\%) underwent

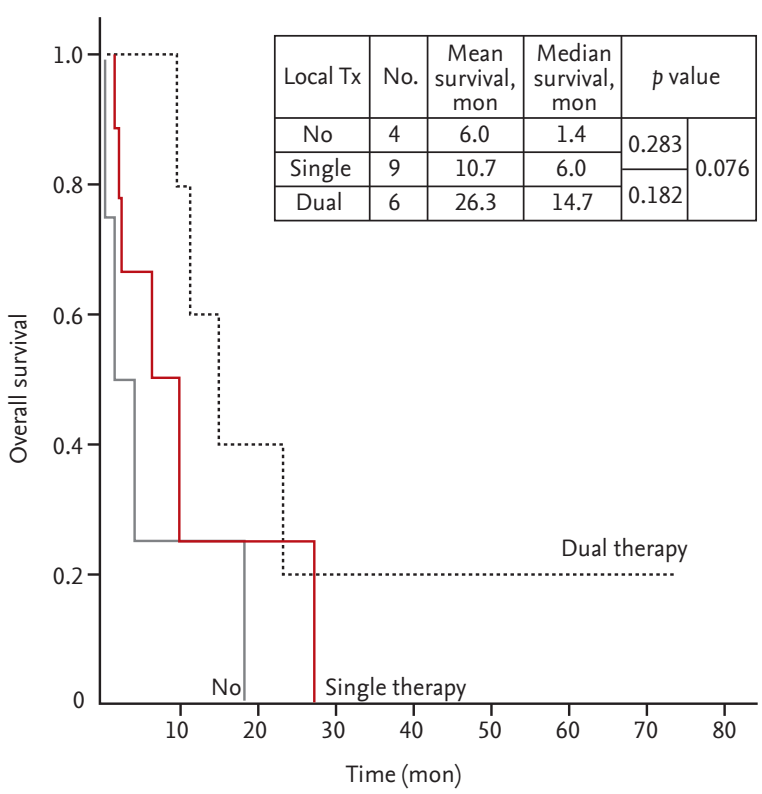

Figure 3. The Kaplan-Meier survival curve in patients with brain metastases from uterine cervical cancer based on the local treatment (Tx) modalities. Single therapy, neurosurgery or radiotherapy; double therapy, neurosurgery and radiotherapy.

local treatment for BM. Single therapy was performed in nine patients (47.4\%); dual therapy with neurosurgery and radiotherapy was performed in six patients (31.6\%).
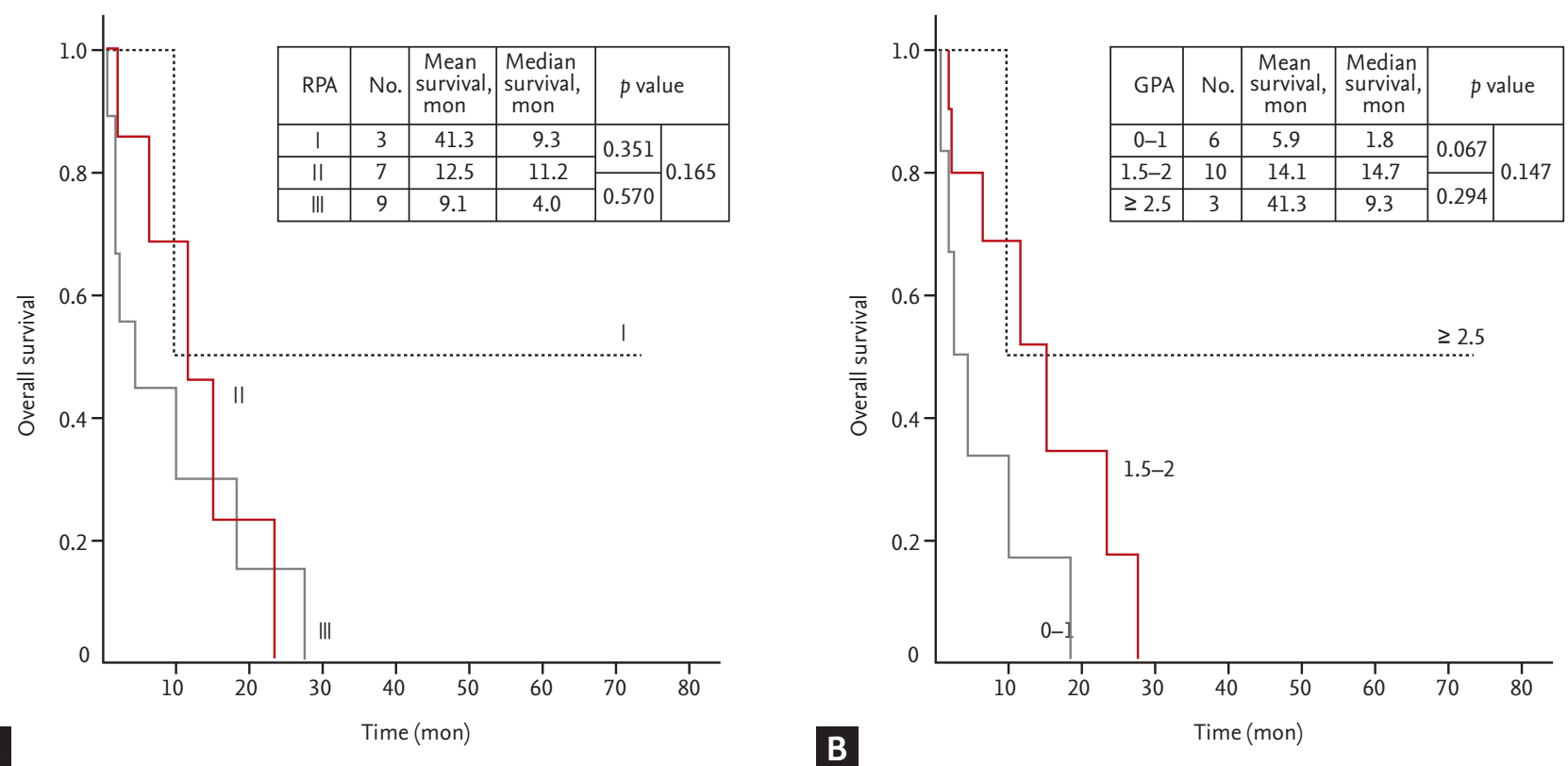

Figure 4. (A) The Kaplan-Meier survival curves according to the recursive partitioning analysis (RPA) class for patients with uterine cervical cancer. (B) The Kaplan-Meier survival curves according to the graded prognostic assessment (GPA) for patients with uterine cervical cancer. 
Thirteen patients $(68.4 \%)$ underwent systemic chemotherapy after BM. Two patients had a solitary BM and were treated with neurosurgery followed by radiotherapy and chemotherapy.

The median and mean OS of all patients was 9.6 months (95\% confidence interval [CI], 3.9 to 15.3 ) and 15.4 months (95\% CI, 5.7 to 25.1), respectively. The Kaplan-Meier survival curve in patients with BM from uterine cervical cancer is shown in Fig. 1.

\section{Prognostic factors influencing overall survival in patients with brain metastasis of uterine cervical cancer}

Treatment (vs. palliative care, median 11.2 months vs. 0.3 months, $p<0.001$ ), fewer than three regimens of chemotherapy before BM (vs. $\geq 3$, median 11.2 months vs. 1.8 months, $p<0.013$ ), and chemotherapy after BM (vs. absence, median 14.7 months vs. 1.4 months, $p<0.001$ ) significantly increased the OS time (Fig. 2).

Other factors listed in Table 2 were evaluated. Although other factors did not reach statistical significance, the observed differences are meaningful. KPS $\geq$ 70 (vs. $<70$, median 11.2 months vs. 4.0 months, $p=0.213$ ) and single BM (vs. multiple BM, median 11.2 months vs. 6.0 months, $p=0.157$ ) had numerically higher OS than others. The patients with small cell carcinoma (vs. others, mean 26.8 months vs. 10.5 months, $p=0.351$ ) had a slightly longer mean OS than the patients with other histology. Dual therapy (vs. single therapy, median 14.7 months vs. 6.0 months, $p=0.182$; vs. no therapy, median 14.7 months vs. 1.4 months, $p=0.076$ ) and single therapy (vs. no therapy, median 6.0 months vs. 1.4 months, $p=$ 0.283) were associated with a longer OS time (Fig. 3).

Furthermore, even though there were no significant differences among each scores or classes in both prognostic scoring systems, GPA (1.5 to 2 vs. O to 1 , median 14.7 months vs. 1.8 months, $p=0.067$; and $\geq 2.5$ vs. $\circ$ to 1 , median 9.3 months vs. 1.8 months, $p=0.147$ ) appears to be a better prognostic tool than RPA (II vs. III, median 11.2 months vs. 4.0 months, $p=0.570$; and I vs. III, median 9.3 months vs. 4.0 months, $p=0.165$ ) (Fig. 4).

\section{DISCUSSION}

The present study aimed to evaluate the clinical char- acteristics and prognostic factors influencing OS in patients with BM from uterine cervical cancer using 19 cases. In the literature, BM from uterine cervical cancer was reported to be extremely rare, with only 115 patients documented in a review of 35 papers before 2012 [12]. Recently, Kim et al. [13] provided a clinical analysis of BM in gynecologic cancers, including 19 uterine cancers, 32 ovarian cancers, and 10 cervical cancers. In addition, Hayashi et al. [8] reported 48 patients with the uterine corpus cancer and 33 patients with the uterine cervical cancer. These studies included all gynecologic cancers, not only uterine cervical cancer; most of other relevant studies were case reports. While uterine cervical cancer is the most prevalent cancer among the gynecologic cancers, it tends to have the lowest incidence of $\mathrm{BM}[2,3]$. In addition, it has the longest interval to $\mathrm{BM}$ and the shortest survival time from BM among the gynecologic cancers [13]. Therefore, owing to a recent increase of the occurrence of BM cases, we need to understand the importance of observing BM from uterine cervical cancer for an earlier detection and longer survival.

$\mathrm{BM}$ is considered to be part of a disseminated disease process and their occurrence is a late event in the course of the disease [12]. Therefore, the prevalence of BM from uterine cervical cancer has increased due to the prolonged survival time from the improved treatment [14]. Although median OS among women with advanced uterine cervical cancer with only cisplatin was about 7 months in 1985 , since 2013 , the combination of chemotherapy and target therapy have led to remarkable increase of OS (by more than 10 months) [15]. In our study, the mean time from diagnosis of the primary uterine cervical cancer in all stages to the appearance of $\mathrm{BM}$ was 35.9 months, which is similar to previous reports $[8,13,16]$.

Currently, BM is usually treated with multimodal therapy using neurosurgery, radiotherapy, and chemotherapy [7]. Previous literature reports that, once BM has developed, the survival rate of a patient with uterine cervical cancer is very low. For instance, in a study by Chura et al. [17], the median survival time from diagnosis of $\mathrm{BM}$ of uterine cervical cancer to death was reported to be 2.3 months. Likewise, Hwang et al. [14] reported that the median survival time after diagnosis of BM was 5.9 months. However, in a recent study, Kim et al. [13] revealed that the median survival time after BM was 8.8 
months, which is comparable to 9.6 months found in our study. Owing to the development of treatment modality, the survival time of patients is expected to gradually increase, which brings about the growing need for optimal treatment protocol of BM from uterine cervical cancer.

Clinical characteristics and treatment modalities influencing the survival of patients with BM from uterine cervical cancer have been reported in the literature. Hayashi et al. [8] emphasized that absence of extracranial metastases, number of BM, and combination of surgery and radiotherapy were associated with good prognosis. Likewise, Kim et al. [13] also reported that the presence of solitary BM, small BM, controlled status of primary cancer, good performance status, and combination therapy were significantly associated with a better survival prognosis. Similarly to these studies, the results of the present study suggest that single BM, good performance status, and the combination of neurosurgery and radiotherapy are good prognostic factors. Furthermore, in addition to demonstrating the importance of chemotherapy after BM, our results also show that less resistance to chemotherapy before BM increases the OS time. In BM patients, the blood-brain barrier is breached, resulting in a higher drug concentration from systemic chemotherapy and, potentially, a more favorable response [13].

Small cell carcinoma of the cervix (SCCC) is rare, accounting for $2 \%$ to $5 \%$ of uterine cervix malignancies [18]. The most common histology of uterine cervical cancer with $\mathrm{BM}$ is squamous cell carcinoma; however, the rate of development of BM is reported to be high in small cell carcinoma [12]. Similarly to small cell carcinoma in other body sites, SCCC is highly invasive and spreads to distant organs $[19,20]$, causing a poorer prognosis than other types of uterine cervical cancer; this makes chemotherapy an important component of multimodality treatment. In our study, we had four patients (21.1\%) of SCCC that was the second most common cancer among patients with BM from uterine cervical cancer. Two patients had only solitary and single BM, while others had the presence of extracranial metastasis. Three patients of SCCC received the combination of local treatment and systemic chemotherapy, and, as a result, they had a slightly longer survival time than the patients with other histology.

Among the analyzed patients, illustrative is a case that makes multimodal therapy strongly suggestive for BM from SCCC. A 50-year-old woman presented with vaginal bleeding for 2 weeks, and the pathologic diagnosis was small cell carcinoma. The clinical stage was determined to be IIB, and the patient underwent concurrent chemoradiation followed by a surgery. Four months afterwards, the patient visited the emergency center with complaints of headache and mental change. Magnetic resonance imaging showed a solitary mass in the right frontoparietotemporal lobe, measuring $39 \mathrm{~mm}$, compatible with a metastatic brain tumor. There were no other metastatic sites on the computed tomography scan. The patient was given neurosurgery, whole brain radiotherapy, and then six cycles of chemotherapy. After treatment, her symptoms were resolved and she has survived with no evidence of disease for 5 years.

Numerous previous studies concluded that GPA was more quantitative and prognostic than RPA in many kinds of metastatic cancers [21,22]. Unfortunately, we were unable to demonstrate the usefulness of these tools in uterine cervical cancer; however, our results revealed that GPA was a better prognostic tool than RPA. This also means that the number of BM is an important prognostic factor, as that is the major difference between the two systems. In order to better define the prognosis of various cancer patients, a number of studies have proposed different disease specific-GPA [23]. For instance, Hayashi et al. [8] proposed uterine-GPA using only two prognostic factors, the number of BM and the existence of extracranial metastasis that are of good prognostic significance. Therefore, prospective randomized studies are needed in the future to find more efficient prognostic factors and develop disease-specific prognostic scoring system in uterine cervical cancer.

The limitations of the present study are related to the inherent biases in a retrospective study and the use of small sample size with a rare type of BM. In addition, the patients with a severe condition were less likely to have a complete evaluation, introducing study biases. Notwithstanding these limitations, the present study provides important information about the prognosis of BM patients from uterine cervical cancer. Further studies with larger sample size and of prospective nature are needed for a more comprehensive and comparative analysis.

In conclusion, in the present study, we evaluated the 
clinical characteristics and prognostic factors influencing OS in patients with BM from uterine cervical cancer retrospectively. Our analysis supports that active treatment, less resistance to chemotherapy before BM, and chemotherapy after BM significantly increased the OS time. In addition, meaningful positive differences were observed for single BM, good performance status, histology of small cell carcinoma, and aggressive local treatment. Taken together, these results suggest the necessity of active multimodal treatment including neurosurgery, radiotherapy, and chemotherapy for BM of uterine cervical cancer with single BM, good performance status, histology of small cell carcinoma, and a better GPA. In addition, observing BM from uterine cervical cancer, regardless of symptoms and the absence of extracranial metastasis, is important for early detection, aggressive multimodal treatment, and a better survival rate.

\section{KEY MESSAGE}

1. The results of the present study suggest active multimodal treatment including neurosurgery, radiotherapy, and chemotherapy for brain metastasis (BM) of uterine cervical cancer with single BM, good performance status, histology of small cell carcinoma, and a better graded prognostic assessment.

2. Observing BM from uterine cervical cancer, regardless of symptoms and the absence of extracranial metastasis, is important for early detection, aggressive multimodal treatment, and a better survival rate.

\section{Conflict of interest}

No potential conflict of interest relevant to this article was reported.

\section{REFERENCES}

1. Torre LA, Bray F, Siegel RL, Ferlay J, Lortet-Tieulent J, Jemal A. Global cancer statistics, 2012. CA Cancer J Clin 2015;65:87-108.

2. Jung KW, Won YJ, Oh CM, et al. Cancer statistics in Korea: incidence, mortality, survival, and prevalence in 2014.
Cancer Res Treat 2017;49:292-305.

3. Sato Y, Tanaka K, Kobayashi Y, et al. Uterine cervical cancer with brain metastasis as the initial site of presentation. J Obstet Gynaecol Res 2015;41:1145-1148.

4. Ogawa K, Yoshii Y, Aoki Y, et al. Treatment and prognosis of brain metastases from gynecological cancers. Neurol Med Chir (Tokyo) 2008;48:57-62.

5. Bindal RK, Sawaya R, Leavens ME, Lee JJ. Surgical treatment of multiple brain metastases. J Neurosurg 1993;79:210-216.

6. Teke F, Tunc SY, Teke M, et al. The impact of the stage and tumor size on rare brain metastasis of cervical cancer. Turk Neurosurg 2016;26:818-823.

7. Richards GM, Khuntia D, Mehta MP. Therapeutic management of metastatic brain tumors. Crit Rev Oncol Hematol 2007;61:70-78.

8. Hayashi N, Takahashi H, Hasegawa Y, et al. A nationwide multi-institutional retrospective study to identify prognostic factors and develop a graded prognostic assessment system for patients with brain metastases from uterine corpus and cervical cancer. BMC Cancer 2017;17:397.

9. Venur VA, Ahluwalia MS. Prognostic scores for brain metastasis patients: use in clinical practice and trial design. Chin Clin Oncol 2015;4:18.

10. Gaspar L, Scott C, Rotman M, et al. Recursive partitioning analysis (RPA) of prognostic factors in three Radiation Therapy Oncology Group (RTOG) brain metastases trials. Int J Radiat Oncol Biol Phys 1997;37:745-751.

11. Sperduto CM, Watanabe Y, Mullan J, et al. A validation study of a new prognostic index for patients with brain metastases: the Graded Prognostic Assessment. J Neurosurg 2008;109 Suppl:87-89.

12. Piura E, Piura B. Brain metastases from cervical carcinoma: overview of pertinent literature. Eur J Gynaecol Oncol 2012;33:567-573.

13. Kim YZ, Kwon JH, Lim S. A clinical analysis of brain metastasis in gynecologic cancer: a retrospective multi-institute analysis. J Korean Med Sci 2015;30:66-73.

14. Hwang JH, Yoo HJ, Lim MC, et al. Brain metastasis in patients with uterine cervical cancer. J Obstet Gynaecol Res 2013;39:287-291.

15. Tewari KS, Sill MW, Monk BJ, et al. Prospective validation of pooled prognostic factors in women with advanced cervical cancer treated with chemotherapy with/without bevacizumab: NRG Oncology/GOG Study. Clin Cancer 
Res 2015;21:5480-5487.

16. Nasu K, Satoh T, Nishio S, et al. Clinicopathologic features of brain metastases from gynecologic malignancies: a retrospective study of 139 cases (KCOG-G1oo1s trial). Gynecol Oncol 2013;128:198-203.

17. Chura JC, Shukla K, Argenta PA. Brain metastasis from cervical carcinoma. Int J Gynecol Cancer 2007;17:141-146.

18. Satoh T, Takei Y, Treilleux I, et al. Gynecologic Cancer InterGroup (GCIG) consensus review for small cell carcinoma of the cervix. Int J Gynecol Cancer 2014;24(9 Suppl 3):S102-S108.

19. Zivanovic O, Leitao MM Jr, Park KJ, et al. Small cell neuroendocrine carcinoma of the cervix: analysis of outcome, recurrence pattern and the impact of platinum-based combination chemotherapy. Gynecol Oncol 2009;112:590593.
20. Gardner GJ, Reidy-Lagunes D, Gehrig PA. Neuroendocrine tumors of the gynecologic tract: a Society of Gynecologic Oncology (SGO) clinical document. Gynecol Oncol 2011;122:190-198.

21. Tabouret E, Metellus P, Goncalves A, et al. Assessment of prognostic scores in brain metastases from breast cancer. Neuro Oncol 2014;16:421-428.

22. Luo J, Zhu H, Tang Y, et al. Analysis of prognostic factors and comparison of prognostic index scores in patients with brain metastases after whole-brain radiotherapy. Int J Clin Exp Med 2014;7:5217-5225.

23. Sperduto PW, Chao ST, Sneed PK, et al. Diagnosis-specific prognostic factors, indexes, and treatment outcomes for patients with newly diagnosed brain metastases: a multi-institutional analysis of 4,259 patients. Int J Radiat Oncol Biol Phys 2010;77:655-661. 